neosalvarsan, and the sores were examined for treponemata 6 to $8 \frac{1}{2}$ hours after injection. Syphilitic spirochætes were present in 8 of the galyl cases as against 5 of the neosalvarsan cases. No spirochetes could be found in any of the 24 sores the morning following injection. I should like to note here that in two cases treated with an intravenous injection of $1 \mathrm{gm}$. ferrivine, the non-arsenical preparation recommended by Mr. J. E. R. McDonagh, treponemata pallida were easily demonstrated four days after injection. The number of cases is too few to place any reliance on, but they suggest neosalvarsan in the dose used to have a quicker treponemicidal action than galyl.

Clinically, in most cases, galyl seems to be almost as valuable as neosalvarsan, ordinary chancres and ulcerative lesions generally clearing up within ten days, but it must be confessed a few cases a month or so after injection still have the remains of a rash and more rarely an unhealed sore, an event which was exceptional in my experience with neosalvarsan. Still, it can exhibit the same dramatic cures as the older drug. The following case is an instance.

A. C- aged 28. Admitted Nov. 7th, 1915. Contracted syphilis in May, 1915. He had extensive ulcerations of the tongue and mouth, a rupial rash, some of the lesions $1 \frac{1}{2}$ in. in diameter, over the whole trunk and limbs, general lymphadenitis, and anal condylomata. He was wasted, anæmic, and felt "very ill." After $0.4 \mathrm{gm}$. galyl on Nov. 10th the rupial crusts separated in four days, leaving clean granulating surfaces. On the 21 st he was discharged from hospital with all lesions healed, a gain in weight of $7 \mathrm{lb}$, and feeling "quite well."

The above observations point to arsenic as the important element in these drugs. The dose $0.4 \mathrm{gm}$. galyl contains just half as much arsenic as $0.9 \mathrm{gm}$. of neosalvarsan, which probably accounts for the slightly less therapeutic effect and lesser toxicity of galyl. Perhaps the lesser toxicity has something to do with its having four benzene rings to the molecule ; anyhow the following facts are striking. Inorganic arsenic, no ring, very toxic ; one-ringed compounds like atoxyl, less toxic; those with two rings as "606" and "914" still less so; and four-ringed galyl probably the least toxic of the lot.

To conclude, it would seem that though excellent results have been obtained with neosalvarsan and mercury with a month's interval between the intravenous injections, this interval is unnecessarily long in the case of galyl. As the dose recommended has only half the arsenic content of neosalvarsan, the interval could be halved without any more likelihood of the occurrence of cumulative arsenical poisoning (the prevention of which is the object of long intervals), and $I$ am sure if this was done galyl would be as good an agent in the cure of syphilis as neosalvarsan; in fact, the observations of other users of the drug have practically proved it to be so.

Since writing the above I read Lieutenant-Colonel L. W. Harrison and Captain C. H. Mills's interesting communication on ferrivine." Though I only gave two intravenous injections of the drug, before deciding to leave it alone in future, the results confirm their observations. One gm. ferrivine used as supplied without further dilution was given to each of two cases of primary syphilis. Immediately following injection both men suffered from acute collapse, with a livid, sweating skin, scarcely perceptible pulse, and respiratory distress. One of the men vomited and had a reactionary temperature of $103^{\circ} \mathrm{F}$. the same evening. There was, however, no thrombosis at the site of injection. One of these reactions was worse than any immediate result I have seen following over 3000 intravenous injections of neosalvarsan or galyl. (Incidentally eight galyl and three neosalvarsan injections given the same morning with the same apparatus were followed by no unpleasant symptoms.) Spirochætes were demonstrated in the serum from the sores four days after injection (they were not looked for later than this). Ten days later, as no improvement was evident and one of the men had developed a secondary roseola, $0.4 \mathrm{gm}$. galyl was given to each case. The penile sores then healed in a week.

The foregoing article is practically an abstract of a paper to be published in the Journal of the Royal Naval Medical Servioe for July. 1916, to which anyone who is interested is referred for further details.

\section{TWO CASES OF PENETRATING WOUNDS OF THE ABDOMEN INVOLVING THE INFERIOR VENA CAVA.}

\author{
BY D. C. TAYLOR, F.R.C.S.,
}

TEMPORARY CAPTAIN, ROYAL ARMY MIEDICAL CORPS.

THE two following cases of penetrating wounds of the abdomen, complicated by tearing of the inferior vena cava, have recently come under my care.

CASE 1.-Private —- aged 20, admitted to clearing station 12 hours after being hit by a piece of high-explosive shell. Wound of entrance tenth right $\mathrm{rib}$ in posterior axillary line: no exit wound. The patient was pale and had marked abdominal pain, tenderness, and rigidity. No vomiting, pulse 100 ; respirations 28 .

Laparotomy was performed. Warm ether vapour; axillary saline. Incision through right rectus; fair amount of free blood in peritoneum. Right lobe of liver penetrated but not bleeding. A single tear 2 inches long in the greater curvature of the stomach, extending on to the anterior wall near the pyloric end, was found and sutured. The stomach was empty and no contents had been extruded. No lesion of bowel was discovered. A small retroperitoneal hæmatoma was noticed at, and on the right side of the base of the mesentery of the small gut. A rubber tube was passed to the under surface of the liver and the rest of the incision was closed in layers. Pulse at end of operation 120. The pulse began to fail six hours later and the patient became very blanched; he died eight hours after operation.

Necropsy.-No fresh lesion in gastro-intestinal tract was discovered. There was an enormous retroperitoneal hæmatoma on the posterior abdominal wall, but little or no fresh bleeding into the peritoneal cavity. An oval hole of about the area of a threepenny-piece was found in the anterior wall of the inferior vena cava just below the entry of the right renal vein. Careful search failed to find the missile.

CASE 2.-Rifleman - aged 40, admitted to clearing station three and a half hours after being hit by a fragment from a bomb. Entrance wound one inch to right and half an inch above the umbilicus; no exit. A small tag of omentum was extruded. The patient was pale; pulse 120 , abdomen very rigid and tender, had vomited four times.

Laparotomy was performed. Warm ether ; axillary saline four hours after injury; incision through right rectus, excising track of missile; much free blood in peritoneum. Missile had passed through gastro-colic omentum, just missing the colon; two veins were bleeding; ligated. Two large tears in front loop of jejunum were found, one at the mesentery border with a small tear of the mesentery, the other at the free border of the bowel immediately opposite. Both tears were sutured. Much bleeding from the posterior abdominal wall on the right side. The intestines were packed back; $a$ hole was found in the posterior peritoneum, just outside the line of the inferior vena cava, and a considerable retro-peritoneal hæmatoma. The posterior peritoneum was slit up with scissors, when furious bleeding took place from a hole in the anterior wall of the inferior vena cava, at and below the entrance of the right renal vein. The tear was longitudinal and three-quarters of an inch long. Bleeding was checked by placing the tip of the left forefinger in the hole in the vessel. Attempts were made to control the bleeding by compressing the vessel above and below, but failed owing to proximity of the renal vein. A pair of artery forceps was placed on the front of the cava just above the wound to produce a pleating effect and then a series of six other artery forceps were applied from above down, effectually closing the hole, leaving the lumen of the vessel patent but narrowed. The missile was not found. The omentum was then packed around the bodies of the forceps. The abdominal incision was rapidly closed with deep salmon-gut sutures, taking up all the layers; the handles of the artery forceps were left protruding through a gap in the midale of the incision. The pulse at the beginning of the operation was 80 and the blood pressure 140; at the end the pulse was 120 and the blood pressure 80 . On the first day after the operation the patient was surprisingly fit; pulse 112; vomiting at frequent intervals. Rectal salines given; urine normal. On the second day the vomiting stopped. "The patient was taking fluids well ; pulse 80 . The bowels acted naturally; no abdo minal distension; no discharge from wound; urine normal. On the fourth day warm chloroform and ether mixture was given, and the stitches above and below the forceps were removed and the muscles separated. Adhesions had formed a good firm track down to the points of the forceps. The forceps were then removed one by one, and no bleeding took place until the last pair was removed; this bleeding was trivial in amount and easily controlled with a gauze 
plug. Two days later the gauze plug was removed without any further bleeding. The patient did not vomit after the second anæsthetic.

The patient was evacuated to the base on the tenth day, and a month after the injury I heard that he was doing well in England.

I have heard of other cases of wounds involving the inferior vena cava being brought to clearing stations, but I believe they have all ended fatally. The second of my two cases shows that it may be possible to save some of these patients by using forcipressure, or possibly suture in cases where the bleeding can be controlled while the suture is inserted. The reason that these cases do not bleed to death immediately is that the wounds in the posterior peritoneum and the cava are not, or do not remain, directly superimposed.

I am much indebted to Lieutenant-Colonel J. W. Longstaff, R.A.M.C., for permission to publish these notes, and also to Colonel H. M. Rigby, A.M.S., for his advice in the after-treatment of the second case.

\section{ON THE USE OF TUBERCULIN IN GENERAL PRACTICE.}

BY J. LINTON BOGLE, M.D. EDIN.,

LATE TUBERCULOSIS OFFICER UNDER THE COUNTY COUNCIL (PARTS OF LINDSEY), LINCOLNSHIRE.

GraNiEd the two postulates (1) that tuberculin is a useful remedy in the treatment of tuberculosis, and (2) that the use of tuberculin should not be the exclusive rôle of the specialist, but should belong to the armamentarium of the general practitioner, then notes on the main principles of tuberculin administration and hints as to the best practical means of carrying them out should be useful in inducing many medical men in general practice to commence treatment with this valuable remedy in the prevention and cure of the most generally prevalent disease in our country.

The best period to commence a course of treatment is in the early stages of the disease, at a time when only the family medical attendant is aware of its existence, and when a wise use of the proper remedies means all the difference between a vigorous and useful manhood on the one hand and a wasting and lingering malady on the other, followed too often by an early death. My experience as a tuberculosis officer has led me to conclude that amongst medical men generally there is a reluctance to venture on the use of tuberculin, based partly on the knowledge that, carelessly given, it is a potent cause of evil, and partly also on ignorance as to the proper ways and means to begin and to carry out the course of treatment. The notes are designedly simple; they are written for the instruction of those who have not hitherto used this method of treatment.

General Prinoiples of Tubereulin Administration.

Tuberculin, if injected into the healthy, produces no symptoms whatever, but in a tuberculous subject it causes the symptoms of the tuberculin reaction. The theoretical explanation of this reaction is that the tuberculous have present in their tissues a specific antibody or lysin, capable of breaking down the tuberculin molecule with the formation of toxins. After injection these toxins are carried into the general circulation, giving rise to the symptoms of general or focal reaction, and are followed by increase in the blood of antituberculous lysin or antibody, causing a state of tolerance or increased resistance to the effects of the tuberculous poison.

In the administration of tuberculin one of two courses is followed: either (1) it is given in small doses repeated at long intervals, cansing immunisation ; or (2) in initial small doses, advancing rapidly to large and repeated ones, producing immunisation with tolerance. The former is the course taken in glandular tuberculous disease in children, and local tuberculosis; and the latter in autotoxic disease, or pulmonary tuberculosis. When Koch first discovered the tubercle bacillus, and introduced his preparation of "Old Tuberculin," I remember administering the remedy under the advice of a Liverpool consultant. The usual mode of treatment at that time was large and increasing doses, causing severe reactions. As a result, the remedy quickly fell into disrepute. Now the aim of the medical man is the avoidance of reactions, or their production in a very mild degree, as a valuable guide to further treatment.

\section{Varieties of Tubercutin.}

All preparations of tuberculin, whether derived from the human or the bovine bacillus, have similar curative properties. They vary, however, in their strength and character. The preparations I have generally used are P.T.O., or bovine tuberculin; T.A.F., or tuberculin albumose-free ; and T., Koch's Old Tuberculin, belonging to the class of soluble extractives. Also B.E., bacillus emulsion, and P.B.E., bovine bacillus emulsion, belonging to the class of mixed products-i.e., soluble extractions, with the insoluble parts of the tubercle bacillus in addition. Their relative strength is approximately as follows, taking $\mathrm{T}$. as a standard: T. $=1$, T.A.F. $=1$, B.E $=\frac{1}{2}$, P.B.E. $=\frac{1}{2}$, P.T.O. $=1 / 10$.

\section{Preparation of Dilutions.}

The Record tuberculin syringe consists of a glass cylinder with a capacity of 1 c.c., or 16.9 minims, and is divided into tenths-i.e., divisions of 0.1 c.c. The syringe is filled with tuberculin to the first division, the remaining nine divisions are filled with diluent consisting of normal saline with 0.25 per cent. of carbolic acid. This is the first dilution, or Dil. A, and consists of 0.1 c.c. of tuberculin in 1 c.c. of dilution, or 1 in 10. Dil. B, or the second dilution, consists of one division or $1 / 10$ of Dil. A, together with $9 / 10$ of diluent ; it has 0.01 c.c. of tuberculin in 1 c.c., or 1 in 100 . Dil. $C$ in like manner has 0.1 c.c. of Dil. B to 0.9 c.c. of diluent, and $=0.001$ of tuberculin in 1 c.c., or 1 in 1000 . Dil. D has 0.0001 c.c. of taberculin in 1 c.c., and Dil. $\mathbb{E}$ has 0.00001 c.c. in 1 c.c., being respectively 1 in 10,000 and 1 in 100,000 of tuberculin in 1 c.c. of dilution. It is convenient to make these dilutions in bottles containing 10 c.c. (Sir A. E. Wright's vaccine bottles), and these bottles have rubber caps through which the needle can be plunged into the dilution without exposing it to the air.

\section{Mode of Administration.}

The usual method is by subcutaneous injection, although it is also given orally, in which case the dose is usually double that given under the skin; but as there is some doubt whether the gastric juices do not change and weaken the action of the tuberculin, the former method is the one in general use. It is to be preferred for its quick action, its accurate dosage, and also for the guidance given by the local reaction. In non-pulmonary tuberculosis the injection is sometimes made near the site of the diseased gland or joint. In oral administration the preparations used are T.R. or B.E.; they are given in a wineglassful of cold water an hour before a meal.

\section{The Tuberoulin Most Suitable for Children.}

In my experience P.B.E. (the bovine bacillus emulsion), B.E., and, on account of its relative mildness, P.T.O. are the most useful preparations. They give a slow and mild reaction, and it is here that Wright's system of repeated small doses spread over a long period is so valuable. In adults, where pulmonary disease is the usual type, it is advisable to begin with T.A.F. or P.T.O. and to follow, as a second course, with B.E. The first few doses are carefully watched, but usually afterwards they can be rapidly increased until the optimum dose is reached. Some very useful guidance can be obtained by observing carefully the symptoms of the tuberculin reaction. A local reaction, shown by a thickening or swelling in the tissues, with some tenderness, or by redness of the skin, is a warning that the dose is being increased quickly enough, and may soon produce a general reaction. A strong general reaction, shown by raised temperature, sickness, shivering, headache, and malaise, and, focally, by signs of crepitation or hæmorrhage in a diseased lung, points clearly to the dose being too high, and it should be reduced at once.

The main guidance in treatment by tuberculin is to be obtained from a regular observance of the temperature (the opsonic index being left to experts). It should be taken twice daily, at 830 A.M. and 6 P.M., and recorded upon a chart. In all rises of temperature rest is the primary necessity; a week in bed is most beneficial, especially in the early febrile stages of the disease.

\section{Dosage.}

Dosage of tuberculin varies according to the type of the disease. In local tuberculosis in adults an initial dose of 0.00005 , 1/20000 c.c., of P.T.O. or B.E., and in children 